Session 2542

\title{
Use of Static and Predictive Metrics in R, D \& E Management
}

\author{
Donald N. Merino, Ph. D., P. E. \\ Professor of Management and Engineering Management, \\ Stevens Institute of Technology, Hoboken, NJ
}

\section{Introduction}

Metrics are a fundamental part of managing the R, D and E function. Every major R, D and E organization collects and analyzes metrics. Continuous process improvement (CPI) requires metrics. However, as the results of a symposium attended by R, D and E Research Directors of hi-tech companies indicates that no one is satisfied with the metrics they used.

A methodology is presented that shows the relationship between static and predictive metrics and continuous process improvement. A comparison of the pros and cons of static and predictive metrics and experiences are presented.

Findings from SATM and Symposium on metrics in R, D and E

A symposium attended by research directors of leading hi-tech companies in the NJ area on the subject of the use of metrics in Research, Development and Engineering (R, D and E) was held. The symposium was sponsored by the Stevens Alliance for Technology Management (SATM)*.

Some of the observation and lessons learned from this symposium are (Merino, 1993):

- Everyone had an extensive set of metrics and had a TQM process for R, D and E.

- No one was satisfied with the metrics they used in R, D \&E.

- There was a heavy reliance on Static metrics.

- There was great interest in Predictive metrics.

In addition, all the companies agreed that for metrics to be effective they must be directly related to the improvement process in which they are embedded. All the companies had Quality Management (QM / TQM) programs for their organizations and in the $\mathrm{R}, \mathrm{D}$ and $\mathrm{E}$ areas they presented. To understand the value of metrics, one must understand how they fit into the QM/TQM, continuous process improvement (CPI) process.

\section{Continuous Process Improvement (CPI) and Metrics}

Continuous Process Improvement (CPI) is one of the absolutes of quality (Juran 1988). CPI requires measurements and measurements require metrics. Figure 1 represents a typical approach to CPI and the role that metrics play.

In 1993, the Stevens Alliance for Technology Management consists of AT \& T, Bell Labs, Allied Signal R \&T, Exxon R \& E, Picatinny Arsenal (ARDEC), Engelhardt (R \& D).

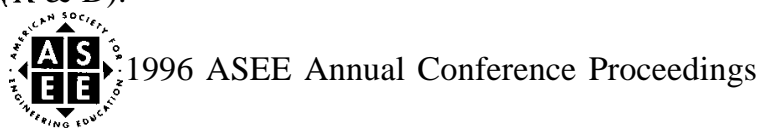


However, the R, D \& E Lab Directors indicated that there are problems associated with the use of metrics. For example, if you measure the time to market of your new product development projects will this result in a reduction of time to market or if careful records are kept of field failure, will the reliability increase? Often metrics are employed in the hope of decreasing time to market and/or reducing costs. Yet these metrics often fall short of delivering the desired results.

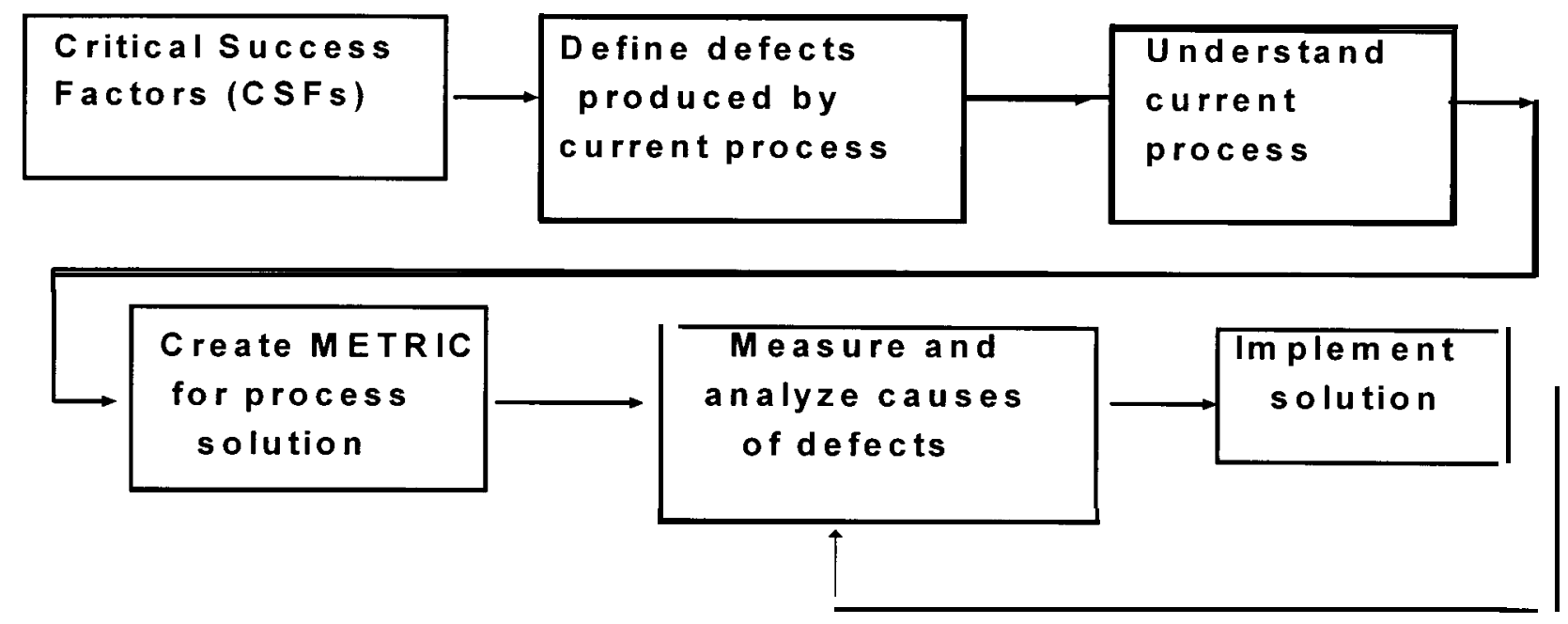

Figure 1 Metrics in the Improvement Process

One conclusion from this experience is that for significant and/or rapid improvements you need to apply metrics in conjunction with focused improvement initiative.

Another conclusion is that the type of metric influences the improvement outcome. Two types of metrics identified were Static and Predictive. In all cases, if metrics are to be really effective they must be coupled with improvement activities. To better understand these conclusions, we must define Static and Predictive metrics.

\section{Types of Metrics : Static and Predictive}

Static metrics measure the results of an entire end-to-end process. They are used to influence the outcome of the next activity, or to make a large improvement or to make relatively infrequent improvements

Static metrics can be applied :

- to the external measurement of projects or programs;

- to motivate change;

- for benchmarking;

- for paradigm shifts; and

- for comparison with other projects or programs.

Predictive metrics measure the quality of sub-process results which are indicative of the outcome of the entire process (often called process metrics). Predictive metrics are used if one wants to influence the outcome of an activity before completion or to make incremental improvements or to make continuous improvements. 
Predictive metrics can be applied to :

- the internal measurement of projects or programs;

- measuring a process continuously and pointing towards change;

- frequent changes; and

- meeting objectives that be reached through constant predictive adjustment.

Predictive metrics are often measured as a relative percent deviation from a target or goal.

\section{Comparison of Static versus Predictive metrics :}

Figure 2 summarizes the difference between Static and Predictive metrics.

\begin{tabular}{|l|l|l|}
\hline & Static & Predictive \\
\hline End Use : & Reactive & Predictive \\
Orientation : & Results & Process \\
Time Frame : & Static & Dynamic \\
\hline
\end{tabular}

Figure 2 Static and Predictive Metrics Compared

Static metrics are useful for identifying benchmarks and for goal settings. Predictive metrics are useful for measuring the difference between current processes and the best-in-breed processes. Static metrics reveal what to do and predictive how to do it.

\section{Misuse of Static and Predictive Metrics:}

Static metrics are used poorly when they are not closely coupled to the process they measure. This leads to imitation without understanding and will result in continuous failure.

Predictive metrics are poorly used on non-critical processes that result in improvement failures. Predictive metrics will not be useful without a visible systematic CPI methodology and in narrowly focused situations.

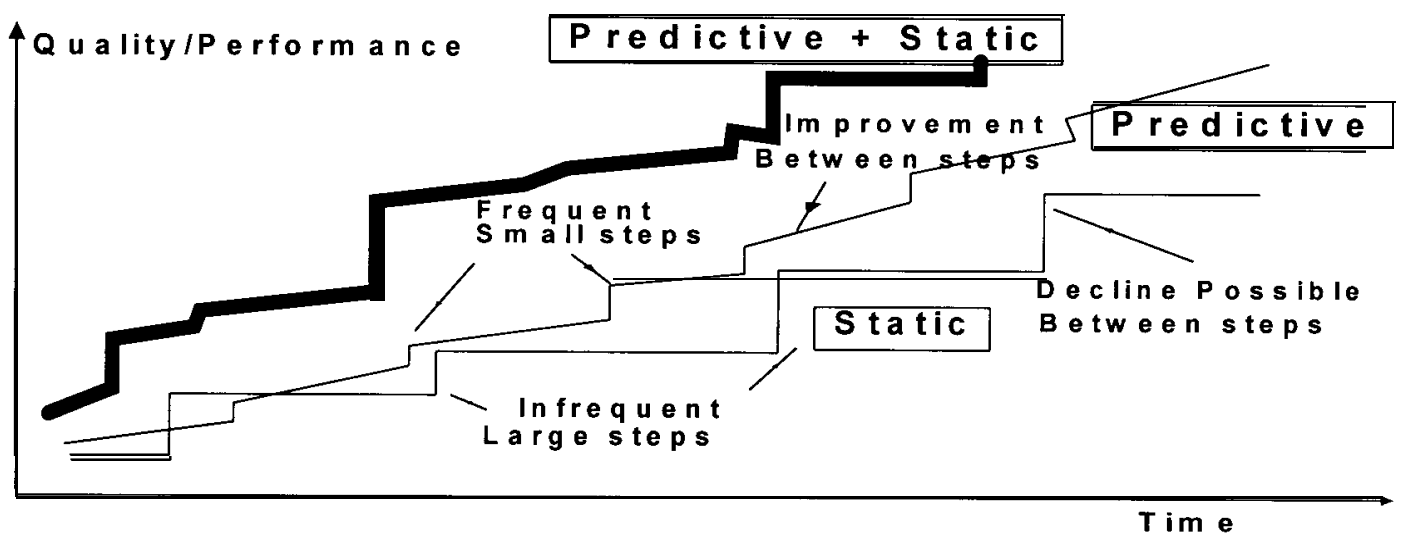

\section{Figure 3 Effective Use of predictive \& Static-Metrics}

\section{Effective Use of Static and Predictive metrics}

As we know from J. Juran (Juran 1988) continuous improvement is really not continuous but rather a series of steps based on specific improvement projects. 
Figure 3 represents graphically how both static and predictive metrics can be used. In this illustration predictive metrics are used to make "mid course" corrections that usually result in smaller improvements while static metrics are used to make end process improvement which are usually more major (e.g. reeingineering).

\section{Examples of Static Metrics}

A static metric example was presented by John Carter (Carter 1992) at the SATM symposium mentioned above. This example of static metrics measured the time to market of various industry groups includes electronics companies, automotive and other.

One example showed that the product development time to market for Japanese consumer electronics companies were about half the time of American companies. A recent article in the Wall Street Journal (Reitman 1995) indicated that the Japanese auto makers' cycle time for developing new cars was about $50 \%$ shorter (18 months versus 24 months) than Ford or Chrysler and more than $100 \%$ shorter (18 months versus 38 months) than GM.

Interestingly, both Ford and Chrysler have previously reorganized their R \& D activities into concurrent engineering multi-disciplinary teams to reduce their previous cycle time (about 60 to 72 months) to 24 months. GM announced a major reorganization of their R, D \& E efforts last year.

\section{Examples of Predictive Metrics}

A good example of a predictive metric is Hewlett Packard's use of product development "report cards". The product development teams grade themselves at least twice during early development to assess their progress. The questions are all indicative of the project's success. Both the mean and standard deviation are calculated. If the mean and standard deviation are ranked low, this indicates problems exist and that if not fixed may result in poor performance.

If the standard deviation is high on any attribute the team tries to narrow the difference via and consultation. Overall, this is a good example of a predictive metric in product development because it provides results early enough to make corrective action possible which would influence the final result.

\section{Summary}

A representative sample of $\mathrm{Hi}$-tech $\mathrm{R}, \mathrm{D} \& \mathrm{E}$ Lab Directors use metrics to measure their process performance but are universally unhappy with the results.

Metrics must be part of a continuous process improvement approach to be useful. It is helpful to distinguish between static metrics that measure end-to-end performance after the fact and predictive metrics that measure sub process that can influence the final outcome.

A combination of static and predictive metrics coupled with a continuous improvement process was presented as a means to effectively monitor R, D \& E progress / performance. Examples of Static and Predictive metrics were presented that illustrated the concepts discussed. 


\section{References}

\section{Carter, John and Graham, Alan}

Metrics and Continuous Improvement

Product Development Consulting,

January 4, 1993

2. Juran, Jim and Gryna, Frank M, Quality Control Handbook - 4th Edition, McGrow-Hill, 1988

\section{Merino D.N.,}

Proceedings : Metrics in R, $D \& E$

Stevens Alliance for Technology Management (SATM),

March 4, 1993

\section{Reitman, Valerie and Simison, Robert $\mathbf{L}$.}

J apanese Car Makers Speed Up Car Making, Wall Street J ournal,

December 29, 1995 pg. B 1

\section{Author's Profile :}

Dr. Donald N. Merino is a tenured full Professor of Engineering Management and Management at Stevens Institute of Technology. He has developed undergraduate and graduate courses and teaches Engineering Economics, Total Quality Management, Decision Analysis and Concurrent Engineering.

$\mathrm{He}$ is the Program Director for the Masters in Technolgy Management (MTM) program. He was founder of the undergraduate program in Engineering Management at Stevens Institute of Technology. He won the Morton Distinguished Teaching Award for full professors. He was PI to develop a concurrent engineering graduate program. His book, "The Selection Process for Capital Projects", was published by John Wiley in their Engineering and Technology Management series.

Dr. Merino received two Centennial certificates from the ASEE in Engineering Economics and Engineering Management. He is past Chair of the Engineering Management Division and Engineering Economy Division of ASEE. Dr. Merino has 25 years of industrial experience in position of increasing managerial responsibility. Since joining acadmia 10 years ago, he has published 18 referred journal articles and over 50 technical reports. He has been an active PI for research progress in Design for Cost / Concurrent engineering; Environmental economics and Medical device development / Economics. 